\title{
Propagation of Electromagnetic Wave through Anisotropic Graded Refractive Index Profile
}

\author{
Arnold Abramov, Lin Luan \\ Kuang-Chi Institute of Advanced Technology, Shenzhen, China \\ Email: qulaser@gmail.com
}

Received November 2013

\begin{abstract}
Propagation of electromagnetic wave (EW) through multilayer structure consisting anisotropic layers with gradient refractive index material is studied theoretically. Analytical solution for linear dependence of refractive index profile is examined. The structure consist the forbidden gaps, which are sensitive to parameters of refractive index profile.
\end{abstract}

\section{KEYWORDS}

\section{Electromagnetic; Refractive Index; Gradient; Layer}

\section{Introduction}

Propagation of EM waves in gradient index medium has been extensively studied in the past century. Materials with refractive indices gradually varying in space are important type of inhomogeneous medium. They can be used in radar devices in the centimeter wavelength range, mobile satellite communications, transformation optics, medical applications, as an optics component to optoelectronic system, etc. In particular in 1944 Luneburg analyzed [1] a more common type of spherical inhomogeneous medium with central symmetry where the focus is situated outside the lens. But the use of Luneburg lenses in practical application hampered by technical complexity of manufacturing the lens with variable index of refraction, which determines their high cost. New opportunities to create graded refractive index (GRIN) medium emerged with the discovery of a new class of artificially structured materials [2-4] called metamaterials which make it possible to achieve both negative effective dielectric permittivity $\varepsilon$ and magnetic permeability $\mu$ parameters in a certain frequency bandwidth. This means the ability to have materials in which refractive index changes from negative (left-handed metamaterials (LHM)) to positive (conventional, right-handed metamaterials (RHM)) values (and vice versa). Propagation of EW in GRIN media studied in $[5,6]$. The choosing of hyperbolic tangent dependence for permittivity and permeability allowed analytical results presented in [5]. Resonant enhancement of EW propagating at oblique incidence in metamaterials, with dielectric permittivity and magnetic permeability linearly changing from positive to negative values, has been predicted and theoretically studied in [6]. In [7] optical properties of one dimensional photonic crystals containing graded materials investigated theoretically. Affect of gradation profiles on photonic band gap engineering was shown.

Anisotropic media is additional tool to manipulate EM wave propagation. The term "anisotropic media" is applied to a medium where refractive index is different in measurement along different axes (is directionally dependent). As a result the induced polarization is not parallel to the electric field, and this factor leads to many optical phenomena such as birefringence effect, conical refraction, optical rotation etc. These and other effects have found wide application, and anisotropic structures are an important and fundamental part of many modern semiconductor and optical devices.

In the present work we study theoretically propagation EM wave through anisotropic layers with linear gradient refractive index. The linear dependence is examined as functions of refractive index profile. We studied influence of graded region and angle of incidence on propagation of EM wave in the structure.

\section{Theoretical Formalism}

We consider propagation of EM waves in a nonmagnetic (we put everywhere for dielectric permeability $\mu=1$ ) 
periodic medium made up of two types of alternating layers: homogeneous and graded layer with space-dependent permittivity. In a case of TE (or H-) polarized wave there are nonzero $H_{z}, E_{x}$, and $E_{y}$ components of electromagnetic field which can be given by expressions:

$$
\begin{gathered}
\vec{H}(\vec{r})=-H(x, y) \vec{z}_{0} \\
\vec{E}(\vec{r})=E(x, y) \cos (\theta) \vec{y}_{0}-E(x, y) \sin (\theta) \vec{x}_{0}
\end{gathered}
$$

And then from the Maxwell equations we obtain for $H$

$$
\frac{d^{2} H}{d x^{2}}+\frac{d^{2} H}{d y^{2}}-\frac{1}{\varepsilon} \frac{d \varepsilon}{d x} \frac{d H}{d x}+\omega^{2} \mu \varepsilon H=0
$$

For homogeneous layer solution of Equation (2) can be represented in the form of plane wave Aexp(ikx) + $B \exp (-i k x)$, where $A$ and $B$ are expansion coefficients to be found.

For GRIN layer we assume that effective dielectric permittivity $\varepsilon_{x}, \varepsilon_{y}$, can vary only in the one direction ( $x$ axes) and that the medium is homogeneous in the $y$ direction. The magnetic field can be written as $H=H(x)$ $\exp \left(i k_{y} y\right)$, where $k_{y}$-wave vector in a y direction and $k_{y}=k_{0} \sin (\theta)=w / c \sin (\theta), \theta$-angle of incidence. Introducing dimensionless length $x \rightarrow x / L$ ( $L$-length of gradient region), wave vector $k=\omega L / c$ we have finally

$$
\frac{d^{2} H(x)}{d x^{2}}-\frac{1}{\varepsilon_{y}} \frac{d \varepsilon_{y}}{d x} \frac{d H(x)}{d x}+\left(k^{2} \varepsilon_{y}-\frac{\varepsilon_{y}}{\varepsilon_{x}} k_{y}^{2}\right) H(x)=0
$$

For fixed value of $\varepsilon_{x}$ and $\varepsilon_{y},=\varepsilon_{0}+b x$ Equation (3) can be solved analytically:

$$
\begin{aligned}
& H_{1}(x)=\sqrt{\varepsilon_{0}+b x} J_{2 / 3}\left(\frac{2}{3} k_{p} \sqrt{b}\left(\frac{\varepsilon_{0}+b x}{b}\right)^{3 / 2}\right) \\
& H_{2}(x)=\sqrt{\varepsilon_{0}+b x} J_{-2 / 3}\left(\frac{2}{3} k_{p} \sqrt{b}\left(\frac{\varepsilon_{0}+b x}{b}\right)^{3 / 2}\right)
\end{aligned}
$$

where $k_{p}=\sqrt{k^{2}-k_{y}^{2} / \varepsilon_{x}}$

Using expressions for EM waves in GRIN and homogeneous layers in transfer matrix technique we can find and analyze frequency dependencies of transmission coefficients.

\section{Results and Discussion}

As follows from Equation (3) anisotropy factor can be ignored for normal incidence. Corresponding results of calculation for TH polarization were studied in detail in [7] for different dependences of $\varepsilon_{y}$. In the present paper we consider oblique incidence and anisotropy factor when $\varepsilon_{x} \neq \varepsilon_{y}$. As it is shown below, both factors can significantly affect transmission spectra.

We consider 1D periodic structure embedded inside an isotropic medium (air). Each period consist of GRIN layer of thickness $d_{1}$ with permittivity $\varepsilon_{y}=\varepsilon_{0}+b x$ and homogeneous layer of thickness $d_{2}$ with permittivity $\varepsilon_{h}=$ 1.5. We used next values: $\varepsilon_{0}=4 . b=3, d_{1} / d_{2}=2$. Figure 1 demonstrates frequency dependences of transmission coefficients for $\varepsilon_{x}=1$ and $\theta=10^{\circ}$ and $60^{\circ}$. It is seen that for oblique incidence both width and position of band gap changes. Increase of incidence angle leads to a more clearly defined and wider band gap. To explain these features we consider a structure of Equation (3). Magnitude

$$
k_{p}=\sqrt{k^{2}-k_{y}^{2} / \varepsilon_{x}}=\omega \sqrt{1-\sin (\theta)^{2} / \varepsilon_{x}}
$$

can be accepted as wave vector of EM wave along propagation direction. On the other hand, we can use estimation of frequency band gap from formula [8]

$$
\frac{\Delta \omega}{\omega} k_{p}=\frac{\Delta n}{n} \int \varepsilon_{y}(x)|\vec{E}|^{2} d x
$$

Because solution for $E$ is Bessel function consisting in argument $k_{p}$, integral in (4) will result to inverse dependence of band fap versus $k_{p}$. Such estimation is evident also from asymptotic: for $k_{p}=0$ propagation of EM wave absence, and formula (4) will give us infinity for frequency width of band gap. As incidence angle increases, $k_{p}$ is decreasing and band gap becomes larger (see Figure 2). It is easy to see from the expression for $k_{p}$ 


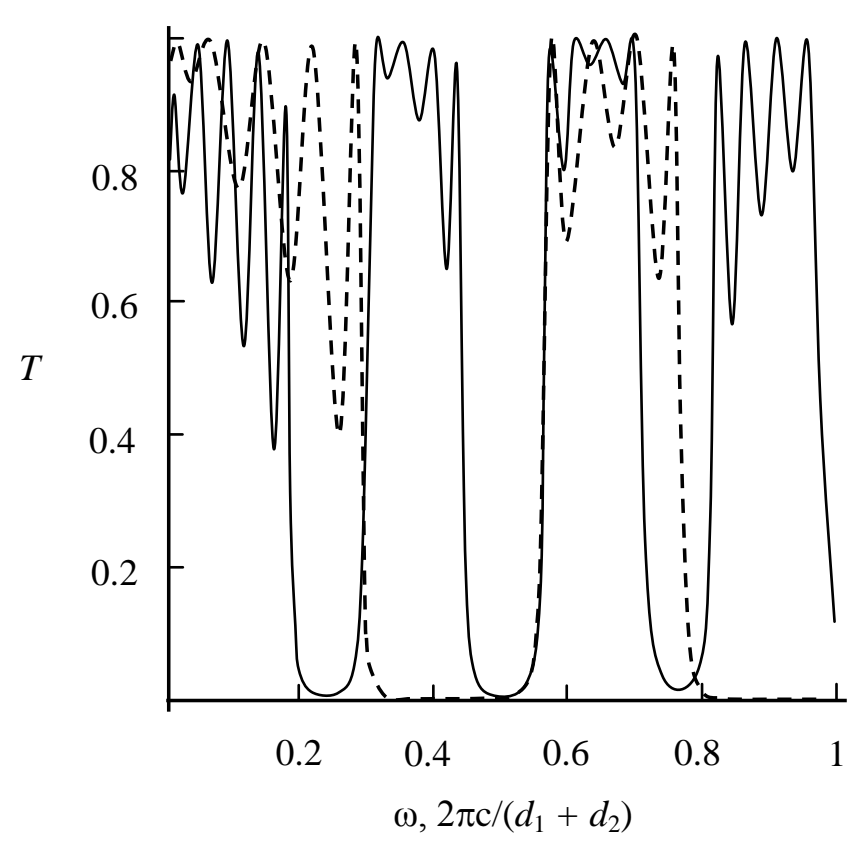

Figure 1. Transmission spectra of the five-period structure for different incidence angles: solid- $\theta=10^{\circ}, \operatorname{dash}-\theta=60^{\circ}$.

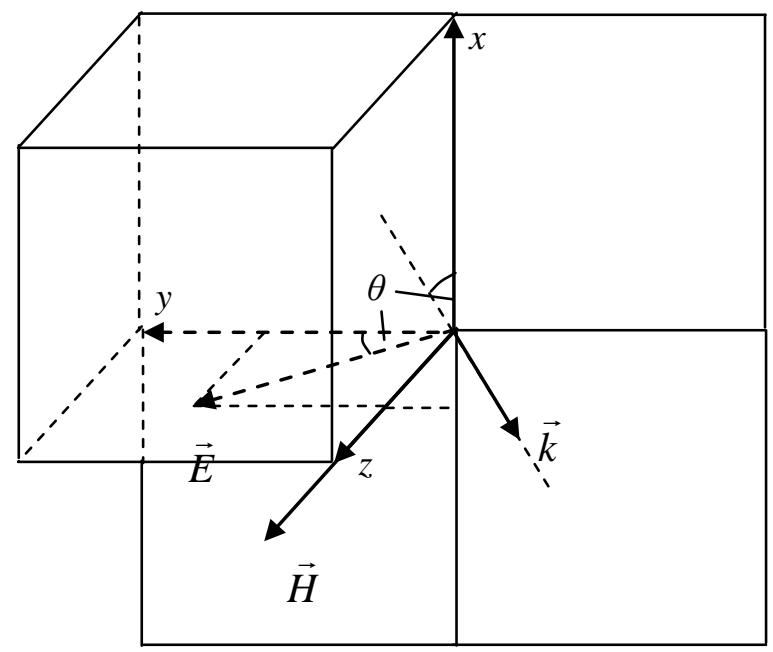

Figure 2. Orientation of the electric and magnetic fields and propagation direction of electromagnetic waves.

that an increase in $\varepsilon_{x}$ is equivalent to reducing the incidence angle, and vice versa. That is, a quite small value of $\varepsilon_{x}\left(\varepsilon_{x}<1\right)$ corresponds to large angles of incidence, for which a larger band gap is formed. On the other hand, a quite big value of $\varepsilon_{X}\left(\varepsilon_{x}>1\right)$ corresponds to a small angles of incidence, for which the spectrum does not change significantly, i.e. it becomes insensitive to a big range of incidence angle.

In conclusion, Maxwell equations solved to find the amplitude of electromagnetic wave propagated through multilayer structure consisting anisotropic layers with gradient refractive index region. Band gaps appearing in transmission spectra can experience significant change as we change incidence angle and (or) anisotropy factor (of dielectric permittivity). The change occurs due to corresponding modification of the wave vector of propagating EM wave.

\section{Acknowledgements}

The work was supported by State Key Laboratory of Meta-RF Electromagnetic Modulation Technology (No. 2011DQ782011), Shenzhen Key Laboratory of Optical and Terahertz Meta-RF (No. CXB201109210101A) and Shenzhen Innovative R\&D Team Program (Peacock Plan) (No. KQE201106020031A), in part by the Rus- 
sian-Ukranian project grant 06-02-12 (U).

\section{REFERENCES}

[1] R. K. Luneburg, “The Mathematical Theory of Optics,” University of California Press, Los Angeles, 1944.

[2] V. G. Veselago, "The Electrodynamics of Substances with Simultaneously Negative Values of $\varepsilon$ and $\mu$," Soviet Physics Uspekhi, Vol. 10, No. 4, 1968, pp. 509-514. http://dx.doi.org/10.1070/PU1968v010n04ABEH003699

[3] E. J. B. Pendry, A. J. Holden, D. J. Robbins and W. J. Stewart, “Low Frequency Plasmons in Thin Wire Structures,” Journal of Physics: Condensed Matter, Vol. 10, No. 22, 1998, pp. 4785-4791. http://dx.doi.org/10.1088/0953-8984/10/22/007

[4] R. A. Shelby, D. R. Smith and S. Schultz, "Experimental Verification of a Negative Index of Refraction,” Science, Vol. 292, No. 5514, 2001, pp. 77-79. http://dx.doi.org/10.1126/science.1058847

[5] M. Dalarsson, Z. Jakšić and P. Tassin, "Exact Analytical Solution for Oblique Incidence on a Graded Index Interface between a Right-Handed and a Left-Handed Material,” Journal of Optoelectronics and Advanced Materials, Vol. 1, 2009, pp. 345-352.

[6] N. M. Litchinitser, A. I. Maimistov, I. R. Gabitov, R. Z. Sagdeev and V. M. Shalaev, "Metamaterials: Electromagnetic Enhancement at Zero-Index Transition,” Optics Letters, Vol. 33, No. 20, 2008, pp. 2350-2352. http://dx.doi.org/10.1364/OL.33.002350

[7] Z.-F. Sang and Z.-Y. Li, “Optical Properties of One-Dimensional Photonic Crystals Containing Graded Materials,” Optics Communications, No. 259, 2006, pp. 174-178.

[8] J. D. Joannopoulos, S. G. Johnson, J. N. Winn and R. D. Meade, “Photonic Crystals: Molding the Flow,” 2nd Edition, Prineston University Press, Prineston, 2008. 